\title{
Inherited XX sex reversal originating from wild medaka populations
}

\author{
A Shinomiya, H Otake, S Hamaguchi and M Sakaizumi \\ Institute of Science and Technology, Niigata University, Niigata, Japan
}

\begin{abstract}
The teleost fish, medaka (Oryzias latipes), has an $X X / X Y$ sexdetermining mechanism. A Y-linked DM domain gene, $D M Y$, has been isolated by positional cloning as the sex-determining gene in this species. Previously, we conducted a field survey of genotypic sex and found that approximately $1 \%$ of wild medaka are sex-reversed ( $X X$ males and $X Y$ females). Here, we performed genetic analyses of nine spontaneous $X X$ sexreversed males to elucidate its genetic basis. In all cases, the $F_{1}$ progeny were all females, whereas $X X$ males reappeared in the backcross $(\mathrm{BC})$ progeny, suggesting that $\mathrm{XX}$ sex
\end{abstract}

reversal is a recessive trait. Although the incidences of sex reversal in the $\mathrm{BC}$ progeny were mostly low, $40 \%$ were males derived from one $\mathrm{XX}$ male. We performed linkage analysis using $55 \mathrm{BC}$ males and located a single major factor, sda-1 (sex-determining autosomal factor-1), controlling sex reversal in an autosomal linkage group. Thus, genes involved in the sex-determining pathway can be isolated from spontaneous mutants in wild populations.

Heredity (2010) 105, 443-448; doi:10.1038/hdy.2010.51; published online 28 April 2010

Keywords: sex determination; sex reversal; sex chromosome; gonad; medaka fish

\section{Introduction}

In vertebrates, primary sex determination occurs in the gonad and the establishment of phenotypic sex outside the gonads is regulated by the gonadal sex. In most mammals, sex determination is controlled genetically by the presence or absence of SRY/Sry (sex-determining region on the $Y$ chromosome), which encodes a putative transcription factor containing a high-mobility groupbox DNA-binding domain (Gubbay et al., 1990; Sinclair et al., 1990; Koopman et al., 1991). Although no ortholog for $S R Y$ has been identified in non-mammalian vertebrates, a Y chromosome-linked gene, DMY (DM domain gene on the $Y$ chromosome, also known as $d m r t 1 b Y)$, was found to be a prime candidate for the sex-determining gene in the teleost medaka (Matsuda et al., 2002; Nanda et al., 2002). DMY encodes a putative transcription factor composed of 267 amino acids. It contains a DNA-binding motif, termed the DM domain, which was originally described as Dsx in Drosophila melanogaster and MAB-3 in Caenorhabditis elegans (Raymond et al., 1998). DMY is expressed during gonadal sex differentiation (Matsuda et al., 2002; Kobayashi et al., 2004) and a loss-of-function mutation of DMY results in XY females (Matsuda et al., 2002; Otake et al., 2006). In addition, testes developed in chromosomally female $(X X)$ medaka when a DNA fragment containing $D M Y$ was introduced as a transgene (Matsuda et al., 2007). These results show that the DMY gene in the medaka behaves dominantly to trigger the differentiation of a testis from a gonadal primordium that would otherwise develop into an ovary. DMY is

Correspondence: $\operatorname{Dr}$ A Shinomiya, Department of Biology, Keio University, 4-1-1 Hiyoshi, Kohoku-ku, Yokohama, Kanagawa, 223-8521, Japan.

E-mail: shinomiya@z7.keio.jp

Received 29 August 2009; revised 20 March 2010; accepted 30 March 2010; published online 28 April 2010 expected to function as a transcription factor; however, the target gene(s) of DMY and other genes implicated in testis and ovary determination are poorly understood.

Analyses of gonadal sex reversal can help uncover the molecular function of a sex-determining gene and identify other genes that might be involved. In our previous study, a survey of 3004 wild fish from 109 localities covering the four genetically divergent groups of the medaka revealed that approximately $1 \%$ of $X X$ (DMY-negative) and $X Y$ (DMY-positive) fish in wild populations were spontaneously sex-reversed individuals (Shinomiya et al., 2004). Examination of the progeny of $23 \mathrm{XY}$ females from the wild revealed that the spontaneous $X Y$ females were fertile and had $\mathrm{Y}$ chromosome-linked mutations (Shinomiya et al., 2004; Otake et al., 2006). All the XY sex reversals were related either to mutations in the open-reading frame of $D M Y$ or to reduced-expression mutations of $D M Y$ (Otake et al., 2006).

In contrast with $X Y$ females, the molecular and genetic basis of the spontaneous $X X$ sex reversals remains unclear. XX males have been reported in some laboratory medaka strains (Aida, 1936; Nanda et al., 2003). Based on crossings between XX females and XX males, Nanda et al. (2003) suggested the existence of a number of autosomal modifier genes affecting sex determination. However, no locus relating to such $\mathrm{XX}$ sex reversals has been identified. In this study, we performed genetic analyses of nine spontaneous $\mathrm{XX}$ males from eight wild populations and report strong evidence for a gene causally related to $\mathrm{XX}$ sex reversal on an autosome, linkage group (LG) 8 .

\section{Materials and methods}

Fish

Nine $D M Y$-negative $(X X)$ males from eight localities in Japan were used (Table 1 ). To obtain $F_{1}$ progeny, each $X X$ male was mated with XX females of an inbred strain, $\mathrm{Hd}$ - 
rR (Hyodo-Taguchi and Sakaizumi, 1993). These Hd-rR females were supplied by a subcenter (Niigata University) of the National Bio-Resource Project (medaka) in Japan. An $F_{1}$ female from each cross was mated with the male parent to obtain backcross (BC) progeny. All fish were raised and maintained under standard conditions $\left(27 \pm 2{ }^{\circ} \mathrm{C}\right.$, with a $14: 10 \mathrm{~h}$ light/dark cycle).

\section{Sexing}

The phenotypic sex was determined from secondary sex characters (shape of dorsal and anal fins; Yamamoto, 1975). The genotypic sex was diagnosed from the presence or absence of the DMY gene by PCR amplification from fin clip DNA using common primers for DMRT1 and DMY (PG17.5, 5'-CCGGGTGCCCAAGTGC TCCCGCTG-3' and PG17.6, 5'-GATCGTCCCTCCACAG AGAAGAGA-3'). PCR conditions were as follows: $5 \mathrm{~min}$ at $95^{\circ} \mathrm{C}$, followed by 30 cycles of $30 \mathrm{~s}$ at $95^{\circ} \mathrm{C}, 30 \mathrm{~s}$ at $55^{\circ} \mathrm{C}$ and $40 \mathrm{~s}$ at $72^{\circ} \mathrm{C}$, followed by $5 \mathrm{~min}$ at $72^{\circ} \mathrm{C}$. The length of the $D M Y$ PCR product was different from that of DMRT1, which allowed us to determine the genetic sex using $1 \%$ agarose gel electrophoresis (Shinomiya et al., 2004).

\section{Genotyping and linkage analysis}

DNA was extracted from the tails of the BC fish using a Wizard Genomic DNA Purification Kit (Promega, Madison, WI, USA) according to the manufacturer's instructions. Genome screening was carried out using 120 expressed sequence tag (EST) markers, which cover the 24 medaka chromosome pairs (LG) (Naruse et al., 2004; M Base, http://earth.lab.nig.ac.jp/ mbase/medaka_top. html; Medaka UT Genome Browser Database, http:// medaka.utgenome.org/). PCR amplification of each EST marker was performed as follows: $4 \mathrm{~min}$ at $95^{\circ} \mathrm{C}$, followed by 12 cycles of $30 \mathrm{~s}$ at $95^{\circ} \mathrm{C}, 1 \mathrm{~min}$ at $64-55^{\circ} \mathrm{C}, 3 \mathrm{~min}$ at $72{ }^{\circ} \mathrm{C}$ and 22 cycles of $30 \mathrm{~s}$ at $95^{\circ} \mathrm{C}, 1 \mathrm{~min}$ at $53{ }^{\circ} \mathrm{C}, 3 \mathrm{~min}$ at $72^{\circ} \mathrm{C}$, with a final elongation step of $3 \mathrm{~min}$ at $72^{\circ} \mathrm{C}$. Polymorphism between Shirone 01 and the Hd-rR strain was identified by length differences of the PCR products, restriction fragment length polymorphisms or heteroduplex polymorphisms using $9 \%$ polyacrylamide gel electrophoresis (Kimura et al., 2004). Marker names, assigned LG, primer sequences and types of polymorphisms are listed in Supplemental Table 1. For each marker, we compared the genotype distributions of the $32 \mathrm{BC}$ males and the expected 1:1 ratio using standard $\chi^{2}$-tests. A simple binomial model was then used for investigating the statistical significance of a departure from the expected 1:1 ratio in the genotyping data of markers on LG 8 and LG 19 in 55 BC males. These markers on LGs 8 and 19 are listed in Supplemental Table 2. The binominal model for assessing the significance of the deviation of the expected 1:1 ratio of homozygotes to heterozygotes was constructed as follows. Under the null hypothesis of no linkage, the probably $(p)$ that a male possesses a homozygous genotype is equal to 0.5 . The probability that $k$ of the 55 males possess a homozygous genotype can be computed from the simple binomial formula:

$$
\operatorname{prob}(k / p)=\left(\frac{55}{k}\right) p^{k}(1-p)^{(55-k)}
$$

Under the alternative hypothesis that a male-inducing locus is linked to a marker locus: $P>0.5$. The maximum likelihood estimate of $p$ under the alternative hypothesis is given simply as $k / 55$. A logarithm (base 10) of odds (LOD) score comparing the hypotheses of linkage and nonlinkage for a particular locus can be computed as $\log _{10}(\operatorname{prob}(k / p=k / 55)-\operatorname{prob}(k / p=0.5))$. Supplemental Table 3 gives relevant probabilities and LOD scores for different outcomes.

\section{Histology}

Gonads of adult fish and larvae were fixed overnight in Bouin's fixative and then embedded in paraffin wax. Cross sections were cut serially at $5 \mu \mathrm{m}$ thickness and stained with hematoxylin and eosin.

\section{Results}

To investigate whether the spontaneous $X X$ sex reversal found in wild populations is a simple genetic trait, we crossed nine wild-derived $X X$ males with $X X$ females of the Hd-rR inbred strain and backcrossed each $F_{1}$ female to its male parent. In all cases examined, the $\mathrm{F}_{1}$ progeny were all female, whereas males appeared in BC progeny (Table 1). The male ratios in the BC were low (1.2-13\%) except for one case, Shirone 01 (40\%).

To confirm testicular development of the Shirone 01 line histologically, we dissected adult fish of the BC line and inspected the gonad under a stereomicroscope. All females examined $(n=73)$ had an ovary and all males $(N=55)$ had a testis. Histology showed that a number of oocytes had developed in the BC ovaries (Figure 1c) and that testicular architecture had formed and spermato-

Table 1 Phenotypic sex of the $\mathrm{F}_{1}$ and BC progeny of the wild-derived XX males

\begin{tabular}{|c|c|c|c|c|c|c|}
\hline \multirow[t]{2}{*}{ Male no. } & \multirow[t]{2}{*}{ Collection site } & \multirow[t]{2}{*}{ Collection year } & \multicolumn{2}{|c|}{$F_{1}^{\mathrm{a}}$} & \multicolumn{2}{|c|}{$B C^{\mathrm{b}}$} \\
\hline & & & Female & Male (\%) & Female & Male (\%) \\
\hline 01 & Shirone, Niigata prefecture & $2001^{c}$ & 70 & $0(0)$ & 81 & $55(40.4)$ \\
\hline 02 & Niitsu, Niigata prefecture & 2004 & 132 & $0(0)$ & 82 & $1(1.2)$ \\
\hline 03 & Nikaho, Akita prefecture & 2003 & 28 & $0(0)$ & 78 & $5(6.0)$ \\
\hline 04 & Tsuruoka, Yamagata prefecture & 2001 & 70 & $0(0)$ & 101 & $5(4.7)$ \\
\hline 05 & Mito, Ibaraki prefecture & 2001 & 40 & $0(0)$ & 23 & $1(4.2)$ \\
\hline 06 & Miyazu, Kyoto prefecture & 2007 & 81 & $0(0)$ & 42 & $3(6.7)$ \\
\hline 07 & Sanda, Hyogo prefecture & 2004 & 70 & $0(0)$ & 46 & $3(6.1)$ \\
\hline 08 & Yokohama, Kanagawa prefecture & 2003 & 88 & $0(0)$ & 94 & $14(13.0)$ \\
\hline 09 & Yokohama, Kanagawa prefecture & 2003 & 64 & $0(0)$ & 135 & $2(1.5)$ \\
\hline
\end{tabular}

${ }^{\mathrm{a}} \mathrm{F}_{1}$ was obtained by crossing between a female of the Hd-rR inbred strain and a wild-derived $X X$ male.

${ }^{b} B C$ was obtained by crossing between a (Hd-rR $\times X X$ sex reversal) $F_{1}$ female and the original $X X$ sex reversal.

${ }^{\mathrm{c}}$ Three other XX males were collected at Shirone in 2001 . The phenotypic sex of the progeny between these males and the $\mathrm{F}_{1}$ females of the 01 male were examined (see text in the Discussion section). 

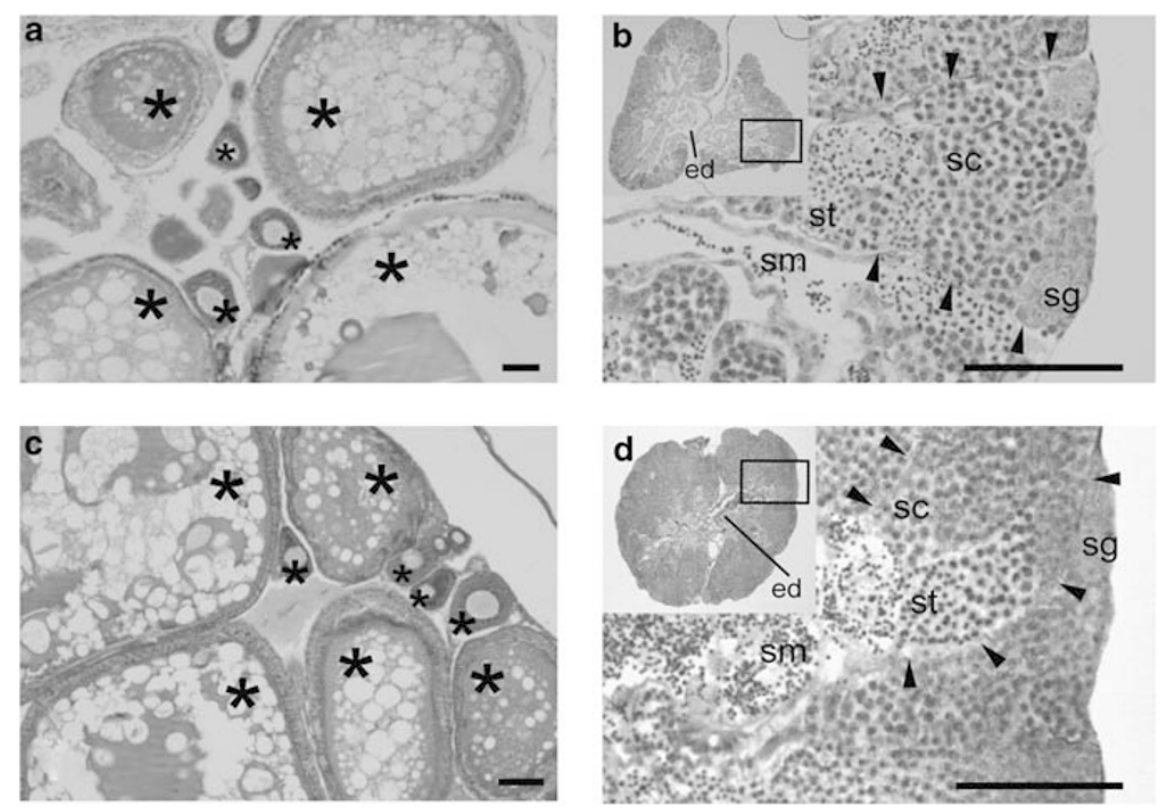

Figure 1 Gonadal histology of adult fish in backcross (BC) progeny of the Shirone 01 male. Gonads in the Hd-rR (a, b) and BC (c, d) adult fish An ovary of BC female (c) and a testis of BC male (d) are similar to a normal ovary (a) and testis (b), respectively. Asterisks indicate various sizes of oocytes at the diplotene stage $(\mathbf{a}, \mathbf{c})$. The black arrowheads show the boundary of a seminiferous tubule (b, d). ed, efferent duct; sc, spermatocytes; sg, spermatogonia; sm, sperm; st, spermatids. Scale bar $=50 \mu \mathrm{m}$.

genesis proceeded normally in the BC testes (Figure 1d) as in normal gonads (Figures $1 \mathrm{a}$ and $\mathrm{b}$ ). We also analyzed the gonads of the BC larvae in earlier stages, which were derived from the crossing of another $F_{1}$ female with the Shirone 01 male, at 10 and 30 days after hatching. At 10 days after hatching, gonads in $88 \%$ of the BC larvae $(N=24)$ were similar to the ovary type seen in normal development. These gonads contained gonial germ cells and numerous oocytes at different developing stages, in which the most advanced oocytes had entered the diplotene stage of meiotic prophase (Figures 2a and c). Conversely, the remaining gonads had gonial germ cells, fewer oocytes at the zygotene or pachytene stages of meiotic prophase and no oocytes at the diplotene stage (Figure 2d). These gonads were similar to the developing testis seen in normal development (Figure 2b). At 30 days after hatching, the $\mathrm{BC}$ gonads could be clearly classified into two types. Eighteen (75\%) BC gonads were normally developing ovaries filled with many oocytes at various stages of the diplotene stage, with oocytes at the zygotene and the pachytene stages and gonial germ cells (Figures $2 \mathrm{e}$ and $\mathrm{g}$ ). In the remaining six gonads $(25 \%)$, we observed gonial germ cells and somatic cells, which formed testis-specific acinous structures: precursors of seminiferous tubules in normal development (Figures $2 \mathrm{f}$ and h) (Kanamori et al., 1985). An efferent duct was present in the central region of the gonads.

To confirm the reappearance of XX male in Shirone 01 progeny, we crossed one of the $B C\left(G_{2}\right)$ males again to an Hd-rR female. The $\mathrm{G}_{3}$ progeny $(N=28)$ were all female. Backcrossing a $\mathrm{G}_{3}$ female with the $\mathrm{BC}$ male produced 13 males $(33 \%)$ in the $\mathrm{G}_{4}$ progeny $(N=40)$. All $B C$ and $G_{4}$ males were confirmed to lack the $D M Y$ gene by PCR on genomic DNA samples.

These histology results and the crossing data indicate that the $D M Y$-negative $\mathrm{XX}$ sex reversal originating from Shirone 01 is a heritable trait. The appearance of males in the $B C\left(G_{2}\right)$ and $G_{4}$ generations and the absence of males in $F_{1}\left(G_{1}\right)$ and $G_{3}$ generations suggest that the Shirone 01-derived alleles at one or a few major loci could cause sex reversal in a homozygous state. Histology showed that the effect of the mutation on the ovarian development appeared around 10 days after hatching.

To search for a gene that caused the XX sex reversal, we first conducted a genome-wide linkage analysis using 120 polymorphic ESTs on a sample of $32 \mathrm{BC}$ males (Supplemental Table 1). The map length of each LG ranged from 38.8 to $98.8 \mathrm{cM}$ and the total map length was $1452.7 \mathrm{cM}$ (Supplemental Table 1), which compares well with the cumulative map length $(1436.8 \mathrm{cM})$ with a panel of 93 female meioses shown by the Medaka UT Genome Browser database (http://medaka.utgenome.org/).

Positions on the LGs showing a correlation between maleness and homozygosity for Shirone 01 alleles are represented graphically in Figure 3 . From the $\chi^{2}$ values alone, a significant deviation $(0.727, P<0.01)$ from the expected 1:1 ratio of Shirone 01 homozygosity to Shirone $01 / \mathrm{Hd}-\mathrm{rR}$ heterozygosity was observed in only two loci on LG 8 (the dark gray area in Figure 3). Two other loci in LG 8 and one locus located in the distal region of LG 19 showed rather a high value $(0.719, P=0.013$; light gray areas in Figure 3). Other loci located on LGs except for LGs 8 and 19 showed lower values than 0.66 $(P=0.077)$.

To better resolve the location of the loci determining maleness, we increased the sample size to 55 males: 10 loci on LG 8 and 3 on LG 19 were analyzed (Supplemental Table 2). After full analysis, deviations from Mendelian expectations of Shirone 01 having a homozygous overrepresentation in BC males remained statistically significant for the loci on LG 8 (Table 2). These results indicate that a major factor that controls $X X$ sex reversal in the medaka is closely linked to Olc01.04h $\left(P=2.37 \times 10^{-6}, \mathrm{LOD}=5.2\right)$. Therefore, we designated the 

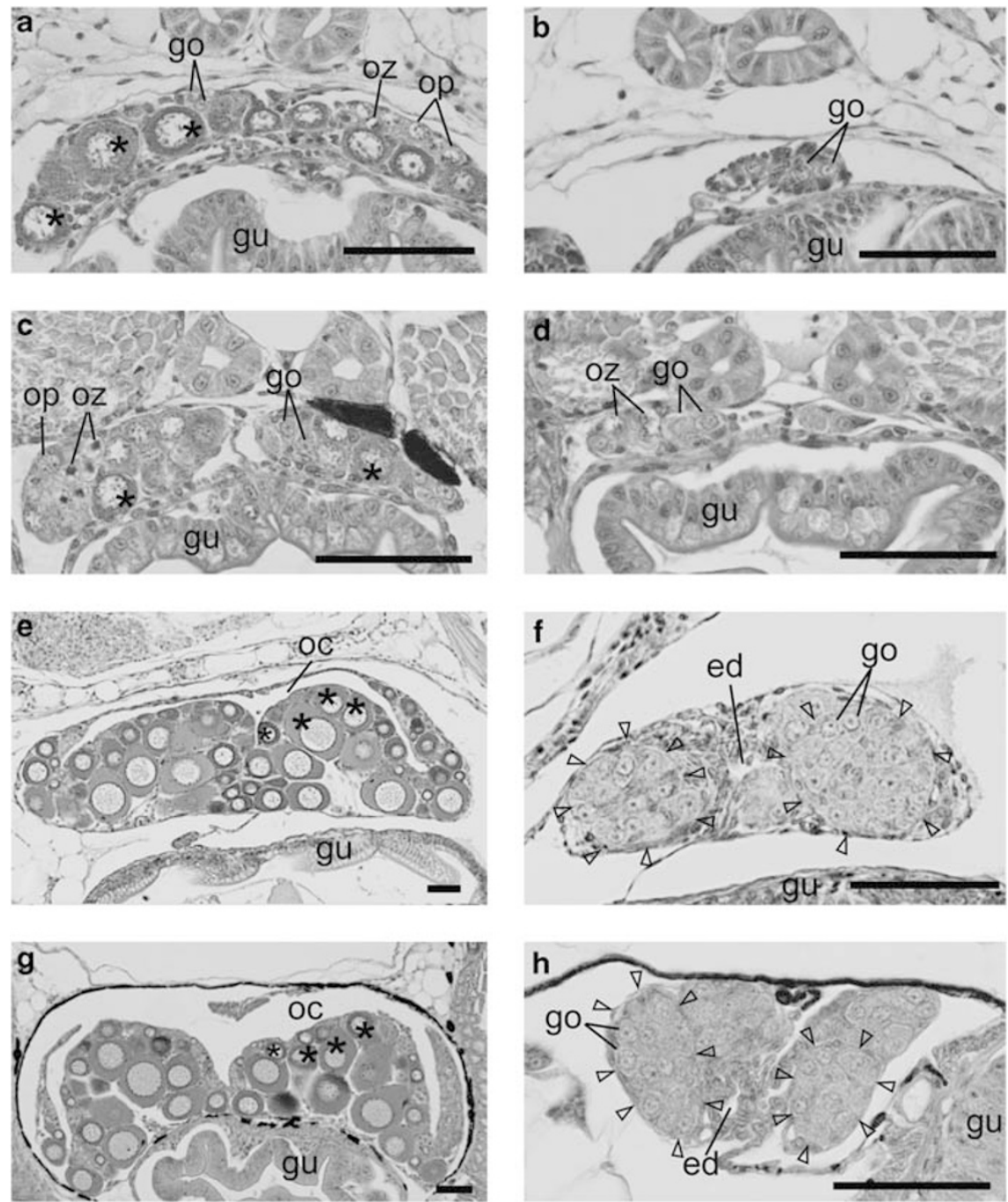

Figure 2 Gonadal histology of BC larvae of the Shirone 01 male. Gonadal histology of the Hd-rR (a, b, e and $\mathbf{f})$ and BC (c, d, $\mathbf{g}$ and $\mathbf{h})$ larvae at 10 days (a-d) and 30 days (e-h) after hatching. An XX (a, e) or XY (b, f) genotype in the Hd-rR larvae. Asterisks indicate various sizes of oocytes at the diplotene stage (a, c, e and $\mathbf{g})$. Open arrowheads indicate acinous structures (f, h). ed, efferent duct; gu, gut; go, gonial germ cells (oogonia or spermatogonia); oc, ovarian cavity; op, oocytes at the pachytene stage; oz, oocytes at the zygotene stage. Scale bar $=50 \mu \mathrm{m}$.

LG 8 locus as sda-1 (sex-determining autosomal factor-1). The distal region of LG 19 was barely significant after full analysis $(P=0.043, \mathrm{LOD}=0.9$; Table 2$)$, ruling out the presence of a strong sex-determining locus.

\section{Discussion}

We have been conducting a broad survey of the genotypic sex of wild medaka since 2001 (Shinomiya et al., 2004) and have found 42 spontaneous DMYnegative XX males to date. In this study, we examined the sex of the progeny of nine wild-derived $X X$ males. To obtain progeny, we used XX females of a laboratoryinbred strain, Hd-rR, in which no spontaneous XX male appeared under typical conditions in our laboratory (Sato et al., 2005). All the $F_{1}$ progeny from the Hd-rR female $(X X)$ and $X X$ males from wild populations developed as female fish, whereas $\mathrm{XX}$ sex reversals appeared in the $\mathrm{BC}$ progeny. Because $\mathrm{BC}$ progeny were raised under the same conditions as the $F_{1}$ progeny, we deduced that the occurrence of $\mathrm{XX}$ sex reversal in $\mathrm{BC}$ depended on genetic factors and that XX sex reversal might be a recessive trait. However, the frequency of the sex reversal was low (1.2-13\%) in eight of the nine cases, which does not indicate a simple monogenic trait. Spontaneous XX sex reversals have also been observed in closed colony breeding stocks: $3.7-15.4 \%$ of males were $\mathrm{XX}$ in six of the eight strains analyzed (Nanda et al., 2003). The incidences of the $X X$ sex reversal varied among the crossings, but there were no $\mathrm{XX}$ males in crossings between different strains (Nanda et al., 2003).

Together with the present results, these results of Nanda et al. (2003) suggest that polygenic factors controlling XX sex reversal might be distributed on the medaka autosomes and/or the $\mathrm{X}$ chromosomes and these seem to be population and strain specific. In the medaka, the phenotypic sex (male or female) essentially depends on the presence or absence of a functional DMY. In addition to this master male-determining gene on the Y chromosome, autosomal or X-chromosomal polymorphic genes might also influence male determination in the medaka. 


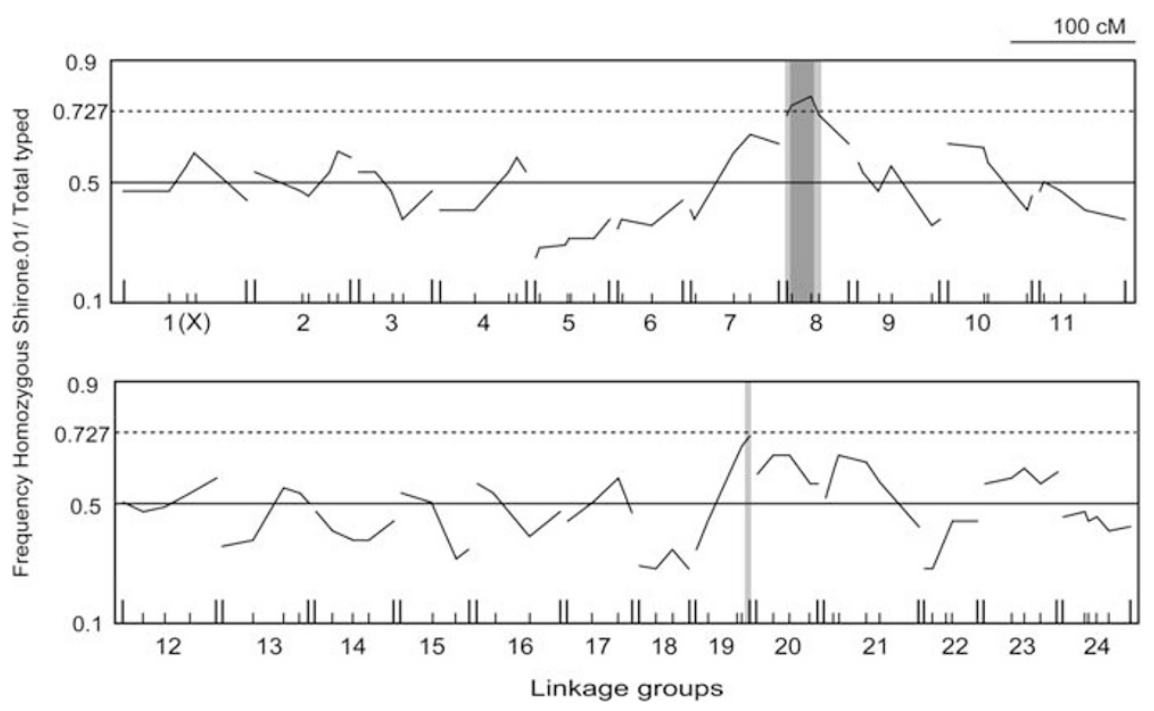

Figure 3 Scanning the genome for linkage in 32 BC males. Plotted is the frequency of homozygous Shirone 01 genotypes among the 32 males at loci spanning the linkage group (LG) 1 through LG 11 (top tier) and LG 12 through LG 24 (bottom tier). Each locus corresponds to the locus listed in Supplemental Table 1. The medaka sex chromosome is LG 1. Map distances were calculated using mapping function of Kosambi (1944). Relative lengths and marker distances along each LG are indicated. The frequency of the Shirone 01/Shirone 01 genotype was expected to be 0.5 (solid line) for chromosomal regions not contributing to sex reversal. Those above 0.727 (dashed line) represent statistically significant departures from expected values using $\chi^{2}$-tests $(P<0.01)$ (dark gray area). Light gray areas indicate loci showing rather high values $(0.719, P=0.013)$

Table 2 Linkage analysis for linkage groups 8 and 19

\begin{tabular}{ccccc}
\hline Linkage group & Distance $(\mathrm{M} b)^{\mathrm{a}}$ & Locus & $\chi^{2} p$ & LOD \\
\hline 8 & 0.5 & Ola04.05c & $1.92 \mathrm{E}-03$ & 2.15 \\
& 4.4 & Ole07.03a & $7.49 \mathrm{E}-04$ & 2.56 \\
& 5.9 & Olb07.09d & $2.72 \mathrm{E}-04$ & 3.01 \\
& 9 & Olc05.03b & $2.91 \mathrm{E}-05$ & 4.03 \\
& 10.3 & Olc01.04h & $2.37 \mathrm{E}-06$ & 5.23 \\
& 11.8 & Olb31.12a & $8.60 \mathrm{E}-06$ & 5.01 \\
& 13.8 & Ola10.09e & $9.22 \mathrm{E}-05$ & 3.49 \\
& 16.2 & Olc09.06c & $2.72 \mathrm{E}-04$ & 3.01 \\
& 22.8 & Ola06.11b & $2.72 \mathrm{E}-04$ & 3.01 \\
& 23.7 & Olb29.07b & $4.63 \mathrm{E}-03$ & 1.79 \\
& 27 & Ola09.09c & $1.04 \mathrm{E}-02$ & 1.45 \\
& & & & \\
& 0 & Sc786.01 & $4.31 \mathrm{E}-02$ & 0.9 \\
& 2.2 & Olb08.01c & $4.31 \mathrm{E}-02$ & 0.9 \\
& 3 & Olb19.06b & $7.96 \mathrm{E}-02$ & 0.67 \\
\hline
\end{tabular}

${ }^{\mathrm{a}}$ The physical distances from an end of the chromosome were based on information from the Medaka UT Genome Browser Database (http://medaka.utgenome.org/).

In comparison with the eight cases of the wild-derived $X X$ males, more $X X$ males reappeared in the Shirone 01 line: all the $F_{1}\left(G_{1}\right)$ and $G_{3}$ progeny were females, whereas 40 and $33 \%$ of $B C\left(G_{2}\right)$ and $G_{4}$ progeny were males, respectively. These results suggest that female-tomale sex reversal is a recessive trait controlled by one or only a few major genes. Linkage analysis on the 55 BC males showed the existence of a major gene, sda-1, which is involved in XX sex reversal. $\chi^{2}$ analysis of the LG 8 allele distribution in the $\mathrm{BC}$ males clearly showed that it was significantly different and that $s d a-1$ was located on the region closely linked to Olc01.04h $\left(P=2.37 \times 10^{-6}\right.$, $\mathrm{LOD}=5.24$; Table 2). The region was localized between two EST markers, Olc05.03b and Olb31.12a, in a region spanning $2.8 \mathrm{Mb}$ (Table 2). These data suggest that the Shirone 01 allele at the sda-1 locus predisposes XX gonads to develop into testicular tissue. The male ratios in $B C\left(G_{2}\right)$ and $G_{4}$ progeny were under $50 \%$ and not all of BC males were Shirone 01 homozygotes at the sda- 1 locus (Figure 3, Supplemental Table 2). These results suggest that there must be multiple minor modifiers that affect the XX sex reversal caused by sda-1. The difference in male ratios seen among $\mathrm{BC}$ offspring from the two $\mathrm{F}_{1}$ females and $\mathrm{G}_{4}$ progeny might depend on the difference of composition of the modifier alleles among the $F_{1}$ and $\mathrm{G}_{3}$ females used in each crossing. These modifiers might be polygenic factors, which would be expected in the other wild-derived XX males and XX males in some laboratory strains. The effects of such modifiers make fine-scale genetic mapping of the $s d a-1$ locus difficult. To remove these effects and to perform fine mapping of the sda-1 locus, we need to establish a Shirone 01-XX/XX strain with a homogenous genetic background. To establish a congenic strain having the $s d a-1$ region from the Shirone 01 on the Hd-rR genetic background, we have started repeating backcrosses of the XX sexreversed males to the Hd-rR inbred females.

The Shirone 01 XX male was found originally in a wild population at Shirone in Niigata prefecture, Japan. We have performed genotypic sexing of wild-caught fish from the same site every year from 2001 to 2006 and found a total of nine males from $322 \mathrm{XX}$ fish. Three of these caught in 2001 were crossed with the Hd-rR strain females, and an $F_{1}$ female from each cross was mated with the BC male offspring of Shirone 01. In all three crosses, the $\mathrm{F}_{1}$ progeny were all females whereas $25-57 \%$ were XX males in the subsequent generation, suggesting that these three XX males had the same recessive mutation at the sda-1 locus as the mutation in Shirone 01 . For the $X X$ males found in subsequent years, it seems that the mutant allele of the sda-1 gene has been maintained in the wild Shirone population through successive generations. 
In the medaka, the sex chromosomes are homomorphic (Matsuda et al., 1998) and sex-reversed XY females and XX males are fully fertile (Yamamoto 1953, 1958). The $Y$ chromosome-specific region in the medaka is no more than $258 \mathrm{~kb}$ in length and $D M Y$ is regarded as the only functional gene in the Y-specific region (Kondo et al., 2006). Thus, the medaka $Y$ chromosome is regarded as equal to the $X$ chromosome except for its sexdetermining function. Aberrant $Y$ chromosomes with a loss-of-function mutation in DMY behaved as $\mathrm{X}$ chromosomes and were maintained in some wild populations (Otake et al., 2008), indicating that functional transformation from the $\mathrm{Y}$ chromosome into the $\mathrm{X}$ chromosome occurs naturally. In this study, we have shown inherited XX sex-reversal from wild populations and identified the sda-1 locus on a medaka autosome. This is one of the major factors controlling spontaneous XX sex reversal. In the Shirone 01 line, successive generations can continue without the $\mathrm{Y}$ chromosome and a pair of autosomes, LG 8, has taken over the role of the sex chromosomes.

In conclusion, we suggest that mutations in genes that interfere specifically with sex determination might not be selected out from wild population in species having undifferentiated sex chromosomes. Such mutations can affect normal sex determination and have the potential to take the place of the current sex-determining gene. Analyses of sex reversals occurring in wild populations can provide us information about the molecular mechanisms of sex determination and will help in understanding unstable sex-determining systems and sex-determining genes in fishes.

\section{Conflict of interest}

The authors declare no conflict of interest.

\section{Acknowledgements}

We are grateful to Dr Kiyoshi Naruse and Dr Hiroshi Mitani for generously providing EST primers and for their technical advice. This work was supported by the Japan Society for the Promotion of Science fellowship (AS), by ExTEND2005 from the Ministry of the Environment of Japan (SH) and by Grants-in-Aid for Scientific Research from the Ministry of Education, Culture, Sport, Science and Technology of Japan (17052007 and 19040009 to $\mathrm{SH})$.

\section{References}

Aida R (1936). Sex reversal in Aplocheilus latipes and a new explanation of sex determination. Genetics 136: 136-153.

Gubbay J, Collignon J, Koopman P, Capel B, Economou A, Münsterberg A et al. (1990). A gene mapping to the sexdetermining region of the mouse $\mathrm{Y}$ chromosome is a member of a novel family of embryonically expressed genes. Nature 346: 245-250.

Hyodo-Taguchi Y, Sakaizumi M (1993). List of inbred strains of the medaka, Oryzias latipes, maintained in the Division of Biology, National Institute of Radiological Science. Fish Biol J Medaka 5: 29-30.

Kanamori A, Nagahama Y, Egami N (1985). Development of the tissue architecture in the gonads of the medaka, Oryzias latipes. Zool Sci 2: 695-706.
Kimura T, Jindo T, Narita T, Naruse K, Kobayashi D, Shin-I T et al. (2004). Large-scale isolation of ESTs from medaka embryos and its application to medaka developmental genetics. Mech Dev 121: 915-932.

Kobayashi T, Matsuda M, Kajiura-Kobayashi H, Suzuki A, Saito $\mathrm{N}$, Nakamoto M et al. (2004). Two DM domain genes, DMY and DMRT1, involved in testicular differentiation and development in the medaka, Oryzias latipes. Dev Dyn 231: 518-526.

Kondo M, Hornung U, Nanda I, Imai S, Sasaki T, Shimizu A et al. (2006). Genomic organization of the sex-determining and adjacent regions of the sex chromosomes of medaka. Genome Res 16: 815-826.

Koopman P, Gubbay J, Vivian N, Goodfellow P, Lovell-badge R (1991). Male development of chromosomally female mice transgenic for Sry. Nature 351: 117-121.

Kosambi DD (1944). The estimation of map distance from recombination values. Ann Eugen 12: 172-175.

Matsuda M, Matsuda C, Hamaguchi S, Sakaizumi M (1998). Identification of the sex chromosomes of the medaka, Oryzias latipes, by fluorescence in situ hybridization. Cytogenet Cell Genet 82: 257-262.

Matsuda M, Nagahama Y, Shinomiya A, Sato T, Matsuda C, Kobayashi T et al. (2002). DMY is a Y-specific DM-domain gene required for male development in the medaka fish. Nature 417: 559-563.

Matsuda M, Shinomiya A, Kinoshita M, Suzuki A, Kobayashi T, Paul-Prasanth B et al. (2007). DMY gene induces male development in genetically female (XX) medaka fish. Proc Natl Acad Sci USA 104: 3865-3870.

Nanda I, Kondo M, Hornung U, Asakawa S, Winkler C, Shimizu A et al. (2002). A duplicated copy of DMRT1 in the sex-determining region of the $Y$ chromosome of the medaka, Oryzias latipes. Proc Natl Acad Sci USA 99: 11778-11783.

Nanda I, Hornung U, Kondo M, Schmid M, Schartl M (2003). Common spontaneous sex-reversed XX males of the Medaka Oryzias latipes. Genetics 163: 245-251.

Naruse K, Tanaka M, Mita K, Shima A, Postlethwait J, Mitani H (2004). Medaka gene map: the trace of ancestral vertebrate proto-chromosomes revealed by comparative gene mapping. Genome Res 14: 820-828.

Otake H, Hayashi Y, Hamaguchi S, Sakaizumi M (2008). The Y chromosome that lost the male-determining function as an $X$ chromosome in the medaka fish, Oryzias latipes. Genetics 179: 2157-2162.

Otake H, Shinomiya A, Matsuda M, Hamaguchi S, Sakaizumi $M$ (2006). Wild-derived $X Y$ sex-reversal mutants in the medaka, Oryzias latipes. Genetics 173: 2083-2090.

Raymond CS, Shamu CE, Shen MM, Seifert KJ, Hirsch B, Hodgkin J et al. (1998). Evidence for evolutionary conservation of sex-determining genes. Nature 391: 691-695.

Sato T, Endo T, Yamahira K, Hamaguchi S, Sakaizumi M (2005). Induction of female-to-male sex reversal by high temperature treatment in medaka, Oryzias latipes. Zool Sci 22: 985-988.

Shinomiya A, Otake H, Togashi K, Hamaguchi S, Sakaizumi M (2004). Field survey of sex-reversals in the medaka, Oryzias latipes: genotypic sexing of wild populations. Zool Sci 21: 613-619.

Sinclair AH, Berta P, Palmer MS, Hawkins JR, Griffiths BL, Smith MJ et al. (1990). A gene from the human sexdetermining region encodes a protein with homology to conserved DNA-binding motif. Nature 346: 240-244.

Yamamoto T (1975). Medaka (Killifish): Biology and Strains. Keigaku: Tokyo.

Yamamoto T (1953). Artificially induced sex-reversal in genotypic males of the medaka (Oryzias latipes). J Exp Zool 123: 571-594.

Yamamoto $T$ (1958). Artificial induction of functional sexreversal in genotypic females of the medaka (Oryzias latipes). J Exp Zool 137: 227-263.

Supplementary Information accompanies the paper on Heredity website (http://www.nature.com/hdy) 